\title{
Determinación de un régimen de caudal ambiental para el río Ñuble considerando actividades recreacionales y requerimientos de hábitat de peces
}

\author{
Environmental flow determination for the River Nuble considering leisure activities and fish \\ habitat requirements \\ Fecha de entrega: 29 de agosto 2017 \\ Fecha de aceptación: 4 de octubre 2018
}

\section{Sofía Toledo ${ }^{1}$ y Enrique Muñoz ${ }^{1,2}$}

${ }^{1}$ Departamento de Ingeniería Civil, Facultad de Ingeniería, Universidad Católica de la Santísima Concepción, Alonso de Ribera 2850, Casilla 297, Concepción, Chile, sofiatoledo@ucsc.cl

${ }^{2}$ Centro de Investigación en Biodiversidad y Ambientes Sustentables CIBAS, Universidad Católica de la Santísima Concepción, Alonso de Ribera 2850, Casilla 297, Concepción, Chile, emunozo@ucsc.cl

El desarrollo hidroenergético genera modificaciones a los patrones hidrológicos en ríos, alterando los ecosistemas acuáticos y el desarrollo de actividades que regularmente ahi se desarrollan. Para evitar alteraciones sobre estos, resulta necesario mantener un caudal mínimo (caudal ecológico), sin embargo, además es necesario minimizar los efectos en el funcionamiento natural del río, sus servicios eco-sistémicos y las actividades que ahi se practican. Sobre esta base se define el caudal ambiental, el cual incorpora aspectos morfológicos y de uso antrópico. En este estudio se analiza y calcula el régimen de caudal ambiental para un tramo del río Nuble considerando aspectos como hidrología y biota, pero también incluyendo en el análisis los usos antrópicos que tradicionalmente se realizan (rafting y kayak). Para calcular el régimen de caudal ambiental se siguieron los lineamientos establecidos en SEA (2016). Se analizaron las componentes de ecología (fauna) y usos antrópicos (actividades recreacionales) mediante el software CASIMIR, utilizando información publicada en el Servicio de Evaluación Ambiental (SEA) y obtenida de entrevistas con los usuarios del río. Como resultado se obtuvo que siguiendo sólo las recomendaciones de SEA (2016) se verán alteradas las actividades recreacionales tradicionalmente realizadas en el río. Por otra parte, si se incluye y cuantifica la necesidad de caudales para el turismo, el caudal ambiental en verano debe ser superior al caudal estimado considerando sólo regulaciones de carácter hidrológico y de funcionalidad de la fauna íctica presente en el río.

Palabras clave: modelación hidrobiológica, caracterización ecológica, caudal ambiental
Hydropower development causes modifications in hydrological patterns in rivers, altering aquatic ecosystems and the activities that are typically carried out there. To avoid direct alterations to rivers, it is necessary to maintain a minimum flow (ecological flow); however, it is also necessary to minimize effects on the natural functioning of the river, its ecosystem services and the activities that are engaged in there. The environmental flow, which incorporates morphological and human-use aspects, is defined based on this foundation. In this study, the environmental flow for a section of the Nuble River is analysed and calculated, not only considering aspects such as hydrology and biota, but also including human activities that have traditionally taken place there (rafting and kayaking). SEA (2016) was used to calculate the environmental flow regime. Ecological and human components were analysed with CASIMIR software using information published in the SEA and obtained through interviews with river users. As a result, it was found that by following only the recommendations of SEA (2016), activities traditionally carried out in the river (tourism) will be altered. In addition, if the need for flows for tourism is included and quantified, the environmental flow should be greater than the flow estimated considering only regulations regarding hydrology and the functioning of the ichthyofauna present in the river.

Keywords: hydrobiological modelling, ecologic characterisation, environmental flow

\section{Introducción}

La modificación de los patrones hidrológicos en ríos a causa del funcionamiento de centrales hidroeléctricas o embalses provoca cambios estructurales y funcionales en los ecosistemas acuáticos. Producto de ello surge la necesidad de mantener un caudal que asegure la estabilidad de dichos sistemas post alteración. El concepto de caudal ecológico, definido como el $20 \%$ del caudal medio mensual, con límite 
máximo en el $20 \%$ del caudal medio anual considerando una estadística de 25 años como mínimo (MMA, 2012), busca proteger los valores ecológicos de los ríos, como la flora y fauna. Sin embargo, en muchos casos, el caudal ecológico corresponde sólo al caudal mínimo que puede mantenerse en un cauce para asegurar la permanencia de estos valores (Jamett y Rodrigues, 2005).

El desarrollo hidroenergético debe estar ligado a regímenes de caudales que permitan la mantención de recursos ecológicos. Diez y Olmeda (2008) sugieren que es posible compatibilizar el aprovechamiento de caudales fluviales para generación hidroeléctrica, manteniendo un estado ecológico global aceptable mediante el cálculo de un régimen de caudal ecológico. Sin embargo, no se hace referencia a la mantención de actividades antrópicas que dependen de los cauces a intervenir. Esto último cobra importancia debido a que la alteración de magnitud y frecuencia de los caudales provocada por el aprovechamiento del recurso, genera alteraciones en el sustrato del lecho del cauce (Reiser et al., 1989), afectando los meso-hábitats existentes y modificando la morfología (Bogan, 1993). En aquellos ríos donde existe un uso antrópico, estas alteraciones generan un impacto directo sobre dichas actividades, cambiando los patrones ecológico-ambientales y los patrones de actividades en torno al río.

En Chile operan más de 100 centrales hidroeléctricas de diferentes características que abastecen al Sistema Interconectado Central SIC, las que se concentran en zonas de cuencas exorreicas. Existen desde minicentrales con una producción menor a $0.5 \mathrm{MW}$, a grandes represas que generan hasta 690 MW. La región del Bío Bío en Chile centro-sur, presenta la mayor capacidad de producción energética del país, con 56 proyectos que pueden aportar un total de 4753 MW al SIC (MinEnergía, 2015). La creación de centrales y minicentrales hidroeléctricas hace prever que se alcanzará un 70\% de producción de energía renovable al año 2025 (MinEnergía, 2016), sin embargo, el excesivo desarrollo hidroeléctrico podría poner en riesgo o eliminar definitivamente los usos recreacionales que tienen los ríos en Chile.

San Fabián de Alico es una comuna turística precordillerana ubicada junto al río Nuble. En sus cercanías existen 2 proyectos hidroeléctricos actualmente en ejecución, Embalse Punilla e Hidroñuble. El proyecto Embalse Punilla está ubicado en el curso medio superior del río Ñuble, $3 \mathrm{~km}$ aguas abajo de la confluencia del río Nuble con el río Los Sauces, y tiene como finalidad asegurar el riego en el valle del río Ñuble en las comunas de Coihueco, Chillán, Ñiquén, San Carlos, San Fabián y San Nicolás, inundando alrededor de 1700 ha, además de generar energía con una producción media anual de 525 GWh (CADE-IDEPE, 2004). Hidroñuble por otra parte, se ubica a $\sim 3.5 \mathrm{~km}$ aguas abajo del proyecto Embalse Punilla. Corresponde a una central de pasada con un caudal de diseño de $100 \mathrm{~m} 3 / \mathrm{s}$ que aportará al SIC una producción media anual de $700 \mathrm{GWh}$.

El Embalse Punilla generará una laminación de caudales en los meses de invierno y un aumento de caudales en los meses de verano. La laminación de caudales alterará componentes ecológicas y antrópicas, como actividades que históricamente se han desarrollado en torno al río Nuble tales como rafting, kayak, pesca deportiva y camping, entre otras. Además de dichos proyectos, existen derechos de agua no consuntivos otorgados para un tercer proyecto, el cual se encuentra en etapa de pre-factibilidad y se emplazaría en un punto cercano a la zona urbana de San Fabián de Alico como se muestra en la Figura 1 (Comité Ambiental Municipal, 2015), lo que podría afectar las actividades de uso antrópico (recreacionales) que se desarrollan en el río y en la comuna, con un potencial impacto sobre el turismo.

El Servicio de Estudio Ambiental SEA (2016) define el caudal ambiental como aquel caudal que permite la mantención de los medios de subsistencia y bienestar de las personas que dependen del ecosistema. Carvajal-Escobar (2008) establece que el caudal ambiental debe prevalecer sobre cualquier otra demanda que impida al río mantenerse en buenas condiciones y asegurar actividades asociadas al ecosistema fluvial. El presente estudio busca definir el caudal ambiental para un tramo de aproximadamente 2 km del río Nuble (Figura 1), incluyendo las actividades antrópicas que tradicionalmente se han desarrollado en este. Con ello, se busca cuantificar los caudales necesarios para evitar impactos sobre la fauna y sobre actividades antrópicas existentes que se desarrollan en torno a este. Para llevar a cabo el análisis se calculó el caudal ambiental siguiendo la guía publicada por SEA (2016) y considerando 
las componentes de hidrología, ecología y de usos antrópicos, con énfasis en las actividades recreacionales asociadas al turismo como el rafting y kayak.

\section{Área de estudio}

El área de influencia se define como el área o espacio geográfico cuyos atributos, elementos naturales $\mathrm{o}$ socioculturales deben ser considerados con la finalidad de definir si el proyecto o actividad genera o presenta algunos de los efectos, características o circunstancias del artículo 11 de la ley, o bien para justificar la inexistencia de dichos efectos, características o circunstancias (SEA, 2017). Para el proyecto que se encuentra en etapa de prefactibilidad, esta corresponde al tramo del río Ñuble de aproximadamente $9 \mathrm{~km}$ comprendidos entre los puntos $1 \mathrm{y}$ 2 de la Figura 1 (Comité Ambiental Municipal, 2015). En dicho tramo se identifican diferentes meso-hábitats, siendo más comunes los meso-hábitats de pozón-rápido. Las zonas de pozón se caracterizan por poseer profundidad alta y velocidades bajas, mientras que los rápidos poseen poca profundidad y velocidades altas. El espaciamiento medio de estos meso-hábitats es de $0.45 \mathrm{~km}$ con una pendiente media del cauce de $0.5 \%$. Además, durante el periodo de estiaje se observan barras a lo largo del cauce debido a la disminución de profundidad del flujo. El régimen hidrológico del río es del tipo pluvio-nival con un caudal medio anual para el tramo de $101 \mathrm{~m}^{3} / \mathrm{s}$. Los mayores caudales se concentran entre mayo y noviembre, producto de un aumento de precipitaciones y posterior derretimiento de nieve (Mendoza, 2015).

Para el cálculo del caudal ambiental y posterior análisis se consideró un tramo de estudio de $2 \mathrm{~km}$ ubicado entre los puntos A y B de la Figura 1. Sobre este tramo se realizó una caracterización morfológica (topográfica) y del sustrato. Dicho tramo se consideró representativo del tramo de influencia, puesto que contiene los meso-hábitats y pendientes que caracterizan todo el tramo de influencia del proyecto.

SEA (2016) indica que es necesario realizar, en el tramo de influencia del proyecto, una caracterización morfológica, ecológica, hidrológica y físico-química de zonas ribereñas, junto con una caracterización de usos antrópicos. Dado que en este estudio sólo se analizan las componentes antrópicas y parte de las componentes ecológicas de la forma indicada

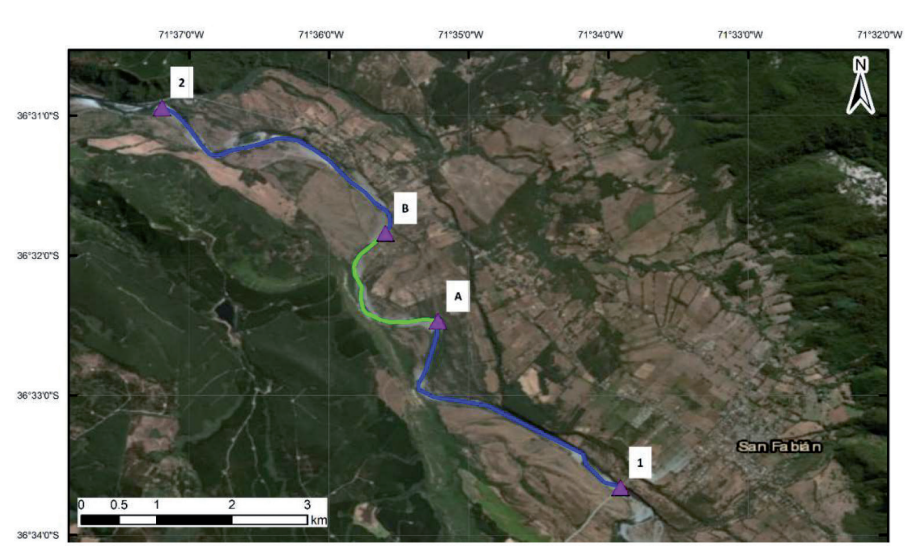

Figura 1: Tramo de influencia del proyecto Embalse Punilla (línea azul entre 1 y 2) y tramo de estudio caracterizado (línea verde entre A y B)

en la SEA (2016), no se realiza una descripción de las componentes relativas a zonas ribereñas y físico-química.

\section{Caracterización de fauna íctica}

De acuerdo a SEA (2016), para determinar el caudal ambiental para centrales hidroeléctricas en el Servicio de Evaluación de Impacto Ambiental SEIA, la caracterización ecológica corresponde al estudio de comunidades y poblaciones de la biota en el ecosistema fluvial. Para desarrollar dicha caracterización se debe conocer la taxonomía, riqueza, abundancia, estructura de tallas y épocas, y sitios de reproducción de especies juveniles y adultas de la fauna íctica presente en el tramo de estudio.

Las especies consideradas se obtuvieron de estudios realizados para los proyectos Embalse Punilla (CADEIDEPE, 2004) cuyos muestreos se realizaron entre los años 2000 y 2002, e Hidroñuble (Illanes y Asociados, 2006) cuyos muestreos se realizaron en el año 2006. Para estos proyectos se realizaron cuatro y una campañas de muestreo respectivamente. En total se encontraron 7 especies ícticas nativas: Diplomystes nahuelbutaensis, Trichomycterus chiltoni, Trichomycterus areolatus, Percilia irwini, Percichthys trucha, Bullockia maldonadoi y Odontesthes Mauleanum y 2 especies introducidas: Oncorhynchus mykiss y Salmo trutta. De las especies introducidas, sólo se consideró en este estudio O. mykiss, dado que $S$. trutta sólo fue hallada en 3 de los 7 puntos de muestreo del proyecto Embalse Punilla.

De acuerdo a lo indicado en los estudios de impacto ambiental de los proyectos Embalse Punilla (2004) e Hidroñuble (2006), de las especies halladas, cuatro 
(P. irwini, P. trucha, O. mauleanum y T. aerolatus) se encuentran en estado de conservación vulnerable, mientras que tres (D. nahuelbutaensis, B. maldonadoi y T. chiltoni) se encuentran declaradas en peligro de extinción.

Respecto a las tallas promedio sólo se tiene información del muestreo realizado para el proyecto Embalse Punilla, en el que se obtuvieron los siguientes datos: $O$. mykiss: $7.5 \mathrm{~cm}$, D. nahuelbutaensis: $4 \mathrm{~cm}, T$. chiltoni: $4 \mathrm{~cm}, T$. areolatus: $5 \mathrm{~cm}, P$ irwini: $4 \mathrm{~cm}$, P. trucha: 12-22 $\mathrm{cm}$ (CADE-IEPE, 2004).

\section{Caracterización hidrológica}

SEA (2016) sugiere desarrollar una caracterización hidrológica describiendo el tipo de régimen del río, magnitud de crecidas ordinarias y periodicidad de fenómenos climatológicos. Para ello se construyeron las curvas de variación estacional mostradas en la Figura 2 para la zona de estudio utilizando los caudales registrados en las estaciones Ñuble en San Fabián y Ñuble en San Fabián 2 (ambas controladas por la Dirección General de Aguas DGA) y corrección de caudales en función del área aportante. Las curvas de variación estacional muestran que el río tiene un régimen pluvio-nival, con un aumento de los caudales entre abril y junio. Posteriormente, entre julio y agosto se observa una leve disminución de caudales y luego un leve aumento de estos entre septiembre y octubre producto del derretimiento de nieve de la parte alta de la cuenca del río Ñuble.

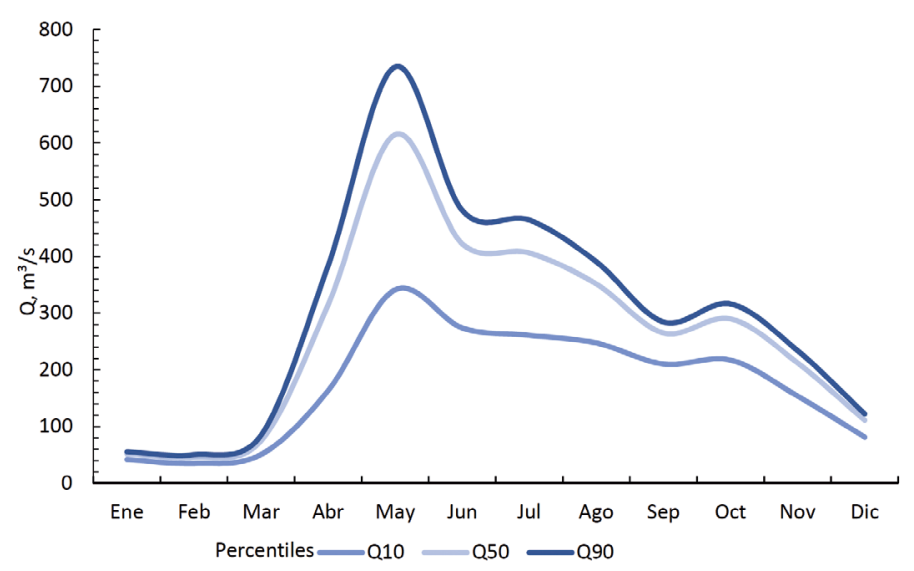

Figura 2: Curva de variación estacional asociada al tramo de estudio para probabilidades de ocurrencia de 10, 50 y $90 \%$ (Q10, Q50 y Q90, respectivamente)

Dado que las crecidas ordinarias corresponden a crecidas pequeñas con periodos de retorno entre 1 y 6 años (SEA,
2016), se realizó un análisis estadístico de caudales máximos instantáneos anuales a partir de 26 años de registros. Se utilizaron las funciones de distribución Pearson, Gumbel, Normal y LogNormal. Para verificar el ajuste entre datos y funciones se realizó la prueba de bondad de ajuste de Kolmogorov-Smirnov. Como resultado se obtuvo que las crecidas de 1 y 6 años se asocian a caudales de 912 y 1797 $\mathrm{m}^{3} / \mathrm{s}$ respectivamente.

\section{Caracterización antrópica}

Mosley (1983) indica que aves, peces y mamíferos no son los únicos que dependen del ecosistema fluvial. El hombre también hace parte de él al realizar diferentes actividades en torno a un río. A su vez menciona 8 factores que describen el comportamiento y apariencia de un río, relacionando factores biológicos y recreacionales, como por ejemplo, características del agua y características morfológicas del río, entre otras. La caracterización de usos antrópicos se requiere para evaluar si existen actividades relevantes en el área de influencia AI y que deban incluirse en el cálculo del régimen de caudal ambiental. En este estudio, se consideraron únicamente actividades in situ de uso sin contacto directo, es decir, aquellas que se desarrollan en el río o en su ribera y en las que el ser humano no toma contacto directo con el agua, pero que son condicionadas por las características hidrológicas del río (SEA, 2016). Entre estas, se consideraron las actividades de rafting y kayak.

SEA (2016) indica que la información para caracterizar las actividades in situ debe ser recopilada mediante la descripción del AI del medio humano. Para ello se contactaron las empresas de turismo Pacífico Andino y Extremo Sur Expediciones, ambas ubicadas en San Fabián de Alico. Dichas empresas realizan actividades de rafting y kayak. En una entrevista, los guías de estas empresas, proporcionaron información cualitativa y cuantitativa sobre las condiciones óptimas en que se desarrollan estas actividades. Cabe destacar que los datos entregados y utilizados para realizar la caracterización antrópica se definieron en base a apreciaciones personales y en función de la experiencia obtenida como guías de empresas de turismo. De acuerdo a lo informado, el rafting y kayak tienen una temporalidad de desarrollo comercial entre los meses de noviembre y febrero, sin embargo, guías 
profesionales y personas expertas pueden realizar bajadas del río durante otoño e invierno. Los requerimientos hidráulicos para desarrollar estas actividades se resumen en la Tabla 1. De acuerdo a lo informado, profundidades y caudales mayores a $160 \mathrm{~m}^{3} / \mathrm{s}$ dificultarían la práctica de kayak y rafting por parte de personas inexpertas, pasando a ser una actividad riesgosa. Por otra parte, caudales inferiores a $80 \mathrm{~m}^{3} / \mathrm{s}$ imposibilita el desarrollo de estas actividades ya que los kayaks y balsas chocarían con el fondo del cauce. Los datos recopilados de las entrevistas coinciden con lo estudiado por Mosley (1983).

Tabla 1: Requerimientos hidráulicos para el desarrollo de rafting y kayak para turismo.

\begin{tabular}{|c|c|c|c|}
\hline & Caudal, $\mathrm{m}^{3} / \mathrm{s}$ & Profundidad, $\mathrm{m}$ & Velocidad, $\mathrm{m} / \mathrm{s}$ \\
\hline Más idóneo & $80-160$ & $0.5-1.5$ & 1 \\
\hline Menos idóneo & $<80 \mathrm{y}>160$ & $<0.5 \mathrm{y}>2$ & $>5$ \\
\hline
\end{tabular}

\section{Metodología}

Para desarrollar el cálculo de caudal ambiental se consideraron las actividades antrópicas de rafting y kayak, y componentes ecológicas asociadas únicamente a la fauna íctica presente en el tramo de estudio.

Para analizar las componentes biológicas (peces) se construyó un modelohidráulico y un modelo hidrobiológico. El modelo hidráulico permitió obtener parámetros hidráulicos a lo largo del río asociados a diferentes caudales, mientras que el modelo hidrobiológico cruza dicha información, con características de preferencias de hábitat de las especies de peces observadas en las cecanías de la zona de estudio, y con necesidades hidráulicas para el desarrollo de actividades antrópicas (turismo).

\section{Modelo hidráulico}

Para construir el modelo hidráulico se realizó una caracterización topográfica del río. Para ello se midieron perfiles transversales a lo largo del tramo de estudio distanciados cada $10 \mathrm{~m}$ aproximadamente. Utilizando el modelo hidráulico HEC-RAS 4.1 (USACE, 2012) en régimen mixto, y considerando que existe un cauce preferente en estiaje, lo que permite simplificar el modelo y evitar considerar al río como multi-cauce, se calculó el eje hidráulico para caudales de $0.5,1,10,20.2,50,80$,
100, 101.1, 150 y 300 m³/s. Se consideró como condición de borde una profundidad crítica debido a los constantes cambios de régimen de sub-crítico a supercrítico y viceversa asociados a la configuración pozón-rápido del río. Como resultado se obtuvo la profundidad de flujo, la elevación de superficie de agua y velocidad media para cada perfil transversal asociado a cada caudal de la modelación.

\section{Modelo hidrobiológico}

El modelo hidrobiológico combina componentes ecológicas e hidráulicas que permiten describir la forma en que un organismo selecciona un microhábitat de acuerdo al índice de idoneidad de hábitat HSI. Este índice representa el hábitat que reúne un conjunto de condiciones en que mejor se desarrolla una especie, y varía entre 0 y 1 , donde 0 es lo menos óptimo (i.e. no existe hábitat adecuado para la especie en análisis) y 1 lo más óptimo (i.e. el 100\% del hábitat disponible es adecuado para la especie en análisis).

Para desarrollar el modelo hidrobiológico se caracterizó la morfología del tramo de estudio en el software CASIMIR (Schneider et al., 2010). A su vez se elaboró una planilla con los datos de altura de superficie de agua para los perfiles transversales y se categorizó el sustrato para cada perfil transversal con valores entre 6 y 9 para tramos con características de rápidos y valores entre 2 y 5 para tramos con características de pozón. Esta información fue obtenida a partir de una foto aérea geo-referenciada adquirida para la zona de estudio. Los valores de categorización del sustrato se obtuvieron de Schneider et al. (2010) del software CASIMIR (Tabla 2).

Tabla 2: Tipos de sustrato utilizados en modelación hidrobiológica según software CASIMIR

\begin{tabular}{|l|c|}
\hline Tipo de sustrato & Índice \\
\hline Material orgánico, detritos & 0 \\
\hline Limo- arcilla & 1 \\
\hline Arena $<2 \mathrm{~mm}$ & 2 \\
\hline Grava fina $2-6 \mathrm{~mm}$ & 3 \\
\hline Grava media $6-20 \mathrm{~mm}$ & 4 \\
\hline Grava grande & 5 \\
\hline Piedras pequeñas & 6 \\
\hline Piedras grandes & 7 \\
\hline Rocas $>20 \mathrm{~cm}$ & 8 \\
\hline Rocas & 9 \\
\hline
\end{tabular}


Para caracterizar los requerimientos o preferencia de hábitat de las especies ícticas T. chiltoni, T. areolatus, y $O$. mykiss se utilizaron curvas de preferencia (EULA, 2000) mientras que las especies $P$. irwini, P. trucha, $B$. maldonado $O$. mauleanum se caracterizaron mediante reglas difusas (García et al., 2011). Las curvas de preferencias son funciones que relacionan la conveniencia para cada especie y etapa de desarrollo, con variables de su hábitat. Estas funciones relacionan valores de cada variable con un índice de idoneidad que varía entre 0 para valoración mínima no aceptable y 1 para idoneidad (Martínez Capel, 2011). En este estudio se analizaron las variables profundidad, velocidad y sustrato.

Las reglas difusas por otra parte, evalúan cuantitativamente expresiones de juicio de expertos de la forma $\mathrm{Si}$ i) velocidad es media, ii) profundidad es media, y iii) sustrato es grande, entonces el hábitat es muy bueno para una especie determinada (Aquaflow, 2015).
Se determinó para cada especie el HSI en función de los caudales analizados. Este índice permite determinar el caudal en que mejor se desarrolla una especie, en sus diferentes estadios. A partir de los resultados es posible obtener el caudal asociado a un $25 \%$ del HSI, el cual, siguiendo el criterio definido por Jowett (1997), se define como aquel HSI mínimo que permite asegurar la mantención de especies.

\section{Resultados y cálculo del caudal ambiental}

Según SEA (2016), el régimen del caudal ambiental debe contener aspectos hidrológicos, hidrobiológicos y de usos antrópicos. De acuerdo al Decreto 14 del Ministerio de Medio Ambiente (MMA, 2012) el caudal ecológico según método hidrológico, corresponde al $20 \%$ del caudal medio mensual, con límite máximo en el $20 \%$ del caudal medio anual, utilizando la estadística hidrológica de los últimos 25 años. La Tabla 3 muestra los caudales medios

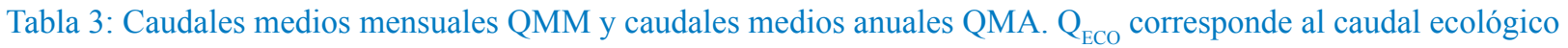

\begin{tabular}{|l|c|c|c|c|c|c|c|c|c|c|c|c|}
\hline Caudal, $\mathrm{m}^{3} / \mathrm{s}$ & Ene & Feb & Mar & Abr & May & Jun & Jul & Ago & Sep & Oct & Nov & Dic \\
\hline QMM & 31 & 24 & 28 & 65 & 158 & 158 & 155 & 153 & 152 & 144 & 99 & 53 \\
\hline $20 \%$ QMM & 6 & 5 & 6 & 13 & 32 & 32 & 31 & 31 & 30 & 29 & 20 & 11 \\
\hline $20 \%$ QMA & 20 & 20 & 20 & 20 & 20 & 20 & 20 & 20 & 20 & 20 & 20 & 20 \\
\hline $\mathrm{Q}_{\mathrm{ECO}}$ & 6 & 5 & 6 & 13 & 20 & 20 & 20 & 20 & 20 & 20 & 20 & 11 \\
\hline
\end{tabular}

Tabla 4: Caudales mínimos obtenidos para asegurar la subsistencia de cada especie, utilizando el criterio del 25\% de HSI (Jowett, 1997).

\begin{tabular}{|c|c|c|c|c|c|c|c|c|c|c|c|c|}
\hline Especie/mes & Ene & Feb & Mar & Abr & May & Jun & Jul & Ago & Sep & Oct & Nov & Dic \\
\hline Percilia irwinii adulto & & & & & & & & 8 & 8 & 8 & 8 & \\
\hline P. irwini juvenil & 4 & 4 & 4 & 4 & 4 & 4 & 4 & & & & & 4 \\
\hline Bullockia. maldonadoi adulto & 8 & 8 & 8 & 8 & 8 & 8 & 8 & 8 & 8 & 8 & 8 & 8 \\
\hline B. maldonadoi juvenil & 9 & 9 & 9 & 9 & 9 & 9 & 9 & 9 & 9 & 9 & 9 & 9 \\
\hline Percichthys trucha adulto & & & & & & 3 & 3 & 3 & & & & \\
\hline P. trucha juvenil & 3 & 3 & 3 & 3 & 3 & & & & 3 & 3 & 3 & 3 \\
\hline Odontesthes mauleanum adulto & & & & & & & 2 & 2 & 2 & 2 & 2 & 2 \\
\hline O. mauleanum juvenil & 3 & 3 & 3 & 3 & 3 & 3 & & & & & & \\
\hline Diplomystes nahuelbutaensis adulto & & & 50 & 50 & & & & & & & & \\
\hline D. nahuelbutaensis juvenil & 20 & 20 & & & 20 & 20 & 20 & 20 & 20 & 20 & 20 & 20 \\
\hline Trichomycterus areolatus adulto & & & & & & & & & 20 & 20 & 20 & 20 \\
\hline Oncorhynchus mykiss adulto & & & & & & & & 25 & 25 & 25 & 25 & \\
\hline O. mykiss juvenil & 7 & 7 & 7 & 7 & 7 & 7 & 7 & & & & & 7 \\
\hline $\mathrm{Q}_{\mathrm{eco}}$ hidrobiológico $\left(\mathrm{m}^{3} / \mathrm{s}\right)$ & 20 & 20 & 50 & 50 & 20 & 20 & 20 & 25 & 25 & 25 & 25 & 20 \\
\hline
\end{tabular}


mensuales obtenidos para el tramo de estudio, y los caudales calculados según MMA (2012).

Utilizando CASIMIR se calcularon los índices de idoneidad de hábitat para cada especie. Utilizando luego el criterio propuesto Jowett (1997) se obtuvieron los resultados indicados en la Tabla 4, para cada especie en estadio adulto y juvenil.

De acuerdo a lo indicado por Extremo Sur Expediciones y Pacífico Andino, kayak y rafting se desarrollan de manera comercial entre los meses de noviembre y febrero con un caudal ideal de entre 80 y $160 \mathrm{~m}^{3} / \mathrm{s}$. Los parámetros de velocidad y profundidad asociados a dichas actividades (Tabla 1) se ingresaron al software CASIMIR, y a partir de este se obtuvo un HSI asociado estas actividades y para los diferentes caudales de análisis. La Figura 3 muestra los resultados obtenidos.

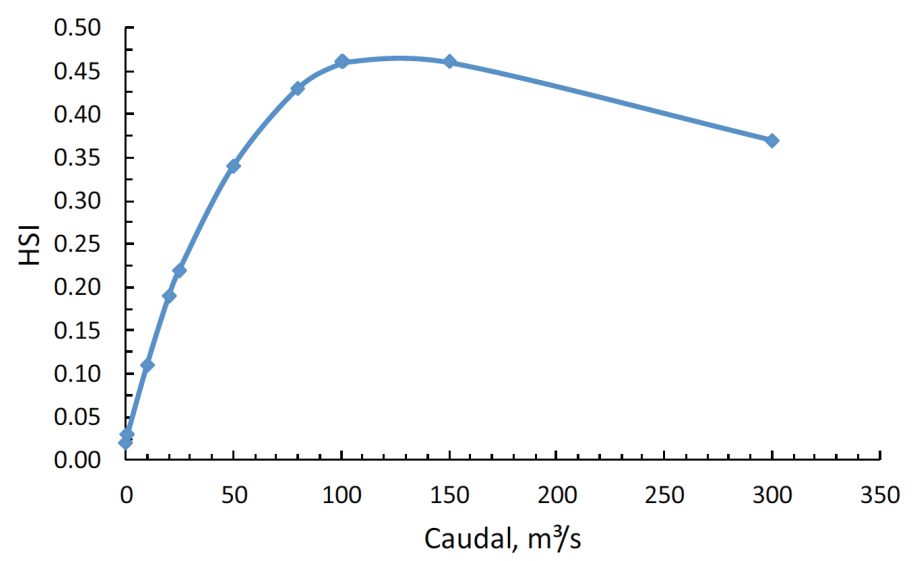

Figura 3: HSI para rafting y kayak considerando los diferentes caudales modelados

La Figura 3 muestra una estabilización de la pendiente para caudales entre 80 y $160 \mathrm{~m}^{3} / \mathrm{s}$. Estos caudales se encuentran dentro del rango adecuado para desarrollar kayak y rafting, sin embargo superan el caudal medio mensual para el tramo de estudio en los meses de enero y febrero ( $\left.\sim 53 \mathrm{~m}^{3} / \mathrm{s}\right)$. Según SEA (2016) el caudal ambiental debe satisfacer tanto los requerimientos de especies como de usos antrópicos. Por lo tanto, el caudal ambiental se obtuvo a partir del cruce de los resultados anteriores y considerando el mayor caudal necesario para cada mes.

La Figura 4 muestra un resumen de los resultados obtenidos y el régimen de caudal ambiental incluyendo usos antrópicos. Adicionalmente, la Tabla 5 muestra los caudales resultantes (la envolvente) del análisis realizado. Es importante mencionar que caudales inferiores a 50 $\mathrm{m}^{3} / \mathrm{s}$ dificultan el desarrollo de actividades antrópicas debido a la disminución de alturas de agua. Como se indica en la Tabla 1, profundidades menores a $0.5 \mathrm{~m}$ impiden el desarrollo adecuado de rafting y kayak, ya que probablemente se formarán islas que interrumpen la conectividad longitudinal del río.

La Figura 5 muestra las profundidades obtenidas del modelo para un caudal de $20 \mathrm{~m}^{3} / \mathrm{s}$. Se muestra que en gran parte del tramo de estudio las profundidades no superan los $0.4 \mathrm{~m}$, profundidades con las cuales no es posible desarrollar las actividades antrópicas mencionadas. Como se observa en la Figura 5, el mayor HSI para rafting y kayak $(\mathrm{HSI}=0.47)$ se obtiene para caudales entre 100 y $150 \mathrm{~m}^{3} / \mathrm{s}$. Para efectos de proponer un caudal adecuado para esta actividad se define un caudal de $50 \mathrm{~m}^{3} / \mathrm{s}$, el cual representa un valor promedio para los meses de enero y febrero, y tiene asociado un índice de idoneidad de 0.35 . Si bien este caudal cumple con los requisitos hidráulicos de velocidad, y profundidad sugeridos por SEA (2016), sólo el 35\% de la superficie utilizada por el río en enero y febrero sería potencialmente útil para las actividades antrópicas. Cabe mencionar que en el caso de actividades antrópicas sólo se consideraron los meses en que estas se desarrollan de manera comercial y por turismo. Los resultados muestran que una disminución de caudales en dichos meses tendrían un impacto sobre el HSI, con un impacto prácticamente proporcional a la reducción de caudales por debajo de 50 $\mathrm{m}^{3} / \mathrm{s}$.

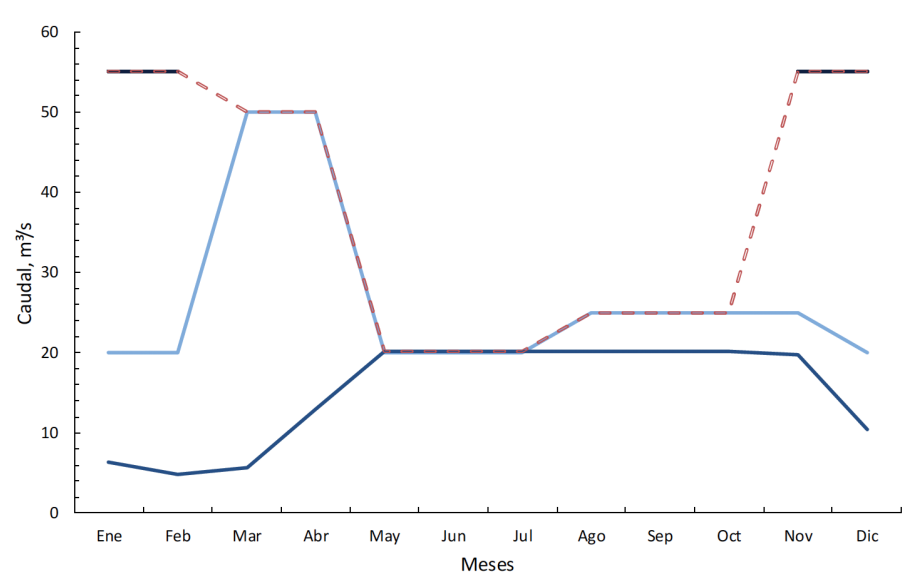

Figura 4: Régimen de caudal ambiental obtenido como la envolvente de los caudales calculados de acuerdo a modelo hidrobiológico e hidrológico. La línea segmentada roja muestra el caudal ambiental obtenido del cruce de información de caudal ecológico (línea azul), caudal hidrobiológico (línea celeste) y caudal para usos antrópicos (línea azul marino) 
Tabla 5: Régimen de caudal ambiental calculado considerando parámetros ecológicos, hidrológicos y antrópicos

\begin{tabular}{|c|c|c|c|c|c|c|c|c|c|c|c|c|}
\hline & Ene & Feb & Mar & Abr & May & Jun & Jul & Ago & Sep & Oct & Nov & Dic \\
\hline $\begin{array}{c}\text { Q } \\
\text { ambiental, } \\
\mathrm{m}^{3} / \mathrm{s}\end{array}$ & 55 & 55 & 50 & 50 & 20 & 20 & 20 & 25 & 25 & 25 & 55 & 55 \\
\hline
\end{tabular}

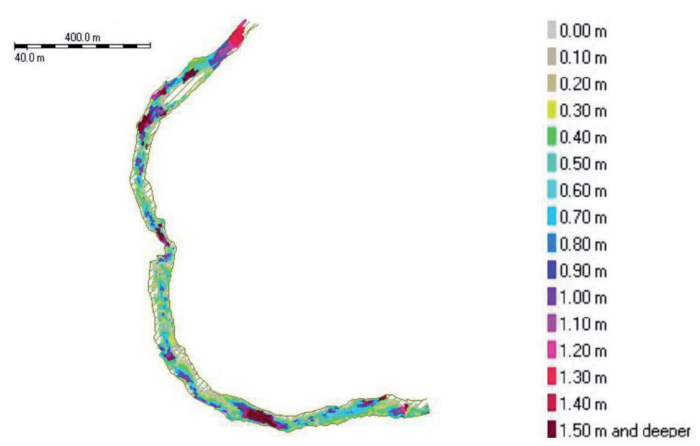

Figura 5: Profundidades en el tramo de estudio para un caudal de $20 \mathrm{~m}^{3} / \mathrm{s}$

SEA (2016) establece criterios de profundidad mínima y velocidad máxima para llevar a cabo actividades antrópicas como rafting y kayak. Sugiere profundidades mínimas de 0.2 y $0.1 \mathrm{~m}$ respectivamente, y una velocidad máxima de $4.5 \mathrm{~m} / \mathrm{s}$, para ambas actividades. La información entregada por SEA (2016), se comparó con la información obtenida del modelo hidráulico para todos los caudales modelados. En la Figura 6 se observa que todos los caudales modelados cumplen con una profundidad mayor a la definida por SEA (2016), sin embargo, profundidades como las indicadas por SEA (2016) resultan inviables para practicar rafting y kayak. Profundidades de $20 \mathrm{~cm}$ o inferiores no permiten la navegación de una balsa y tampoco permiten remar dentro del río, sugiriendo una deficiencia en las recomendaciones establecidas en SEA (2016).

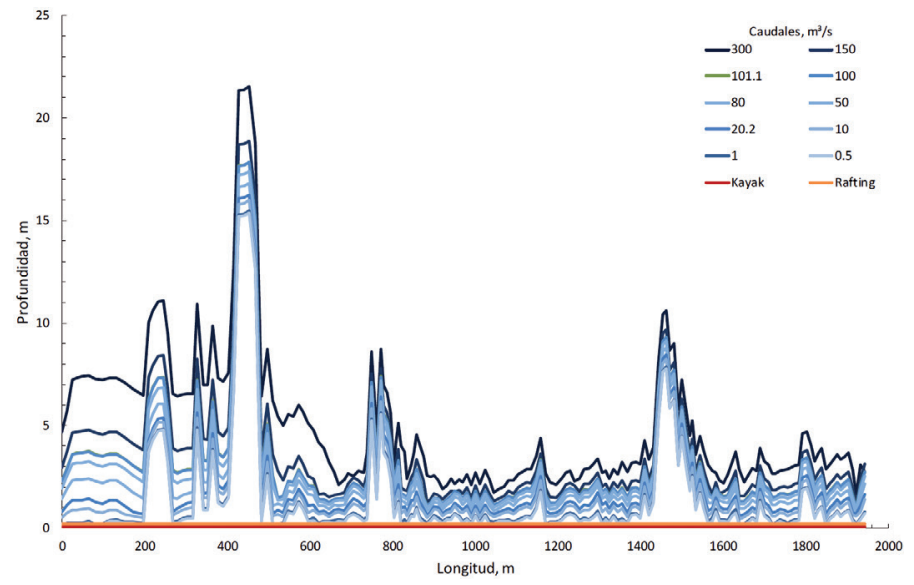

Figura 6: Profundidades asociadas a los caudales modelados para la longitud del tramo de río estudiado partiendo desde aguas abajo

\section{Conclusiones}

Se sugiere un régimen de caudal ambiental para un tramo del río Ñuble en las cercanías de San Fabián de Alico. Este régimen persigue tanto la mantención de especies de fauna íctica, como la mantención de las actividades de usos antrópicos que tradicionalmente se han desarrollado en ese lugar tales como turismo y kayak. En general, los resultados obtenidos y el régimen de caudal ambiental propuesto satisfacen los requerimientos establecidos en SEA (2016). Por el contrario, las profundidades mínimas definidas por SEA (2016) para la mantención de actividades antrópicas como rafting y kayak resultan insuficientes para dicho objetivo, y por lo tanto, considerar sólo los lineamientos indicados en SEA (2016) podría representar una amenaza a la mantención de las actividades de recreación que hoy se realizan en el AI del proyecto. Se estimó que para usos antrópicos se debería contar con un caudal de al menos 50 $\mathrm{m}^{3} / \mathrm{s}$. Dado que en época estival no se cuenta con dichos caudales, se sugiere, al menos mantener el caudal medio mensual natural para favorecer la realización de actividades recreacionales asociadas al turismo. Por otra parte, se estimó que la fauna íctica no se debería ver afectada en caso de desarrollo del proyecto, debido a que las condiciones de oxigenación, velocidad y profundidad del río se mantendrían, principalmente, debido a la morfología del río y de los meso-hábitat presentes, los cuales no sufrirían cambios ante una potencial modificación de los caudales.

\section{Referencias}

Aquaflow (2015). Informe proyecto Central hidroeléctrica La Frontera. Concepción, Chile

Bogan, A.E. (1993). Freshwater bivalve extinctions (Mollusca: Unionoida): a search for causes. American Zoologist 33(6), 599 609

CADE-IDEPE (2004). Línea de base medio biótico proyecto Embalse Punilla. Informe de CADE-IDEPE Consultores en Ingeniería para Dirección de Obras Hidráulicas, Ministerio de Obras Públicas, Gobierno de Chile

Carvajal-Escobar, Y. (2008). Environmental flow regime in the framework of integrated water resources management strategy. Ecohydrology \& Hydrobiology 8(2-4), 307-315 
Comité Ambiental Municipal (2015). Acta Comité Ambiental Municipal de San Fabián de Alico del 9 de diciembre 2015. San Fabián de Alico, Chile

Embalse Punilla (2004). Embalse Punilla VIII Región del Bio Bio. Servicio de Evaluación Ambiental, Gobierno de Chile

EULA (2000). Determinación del caudal mínimo ecológico del Proyecto Hidroeléctrico Quilleco en el río Laja considerando variables asociadas a la biodiversidad y disponibilidad de hábitat. Informe de avance. Unidad de Sistemas Acuáticos, Centro de Ciencias Ambientales EULA-Chile, Universidad de Concepción, Chile

Diez, J.M. y Olmeda, S. (2008). Diseño eco-hidrológico de pequeñas centrales hidroeléctricas: evaluación de caudales ecológicos. Energética 39, 65 - 76

García, A., Jorde, K., Habit, E., Caamaño, D. and Parra, O. (2011). Downstream enviromental effects of dam operations: changes in habitat quality for native fish species. River Research and Aplications 27(3), 312 - 327

Hidroñuble (2006). Central Hidroñuble. Servicio de Evaluación Ambiental, Gobierno de Chile

Illanes y Asociados (2006). Línea de base medio biótico Proyecto Central Nuble de pasada. Informe de Illanes y Asociados para la Dirección de Obras Hidráulicas, Ministerio de Obras Públicas, Gobierno de Chile

Jamett, G. y Rodrigues, A. (2005). Evaluación del instrumento caudal ecológico, panorama legal e institucional en Chile y Brasil. REGA - Revista de Gestão de Água da América Latina 2(1), 83 - 96 Jowett, I.G. (1997). Instream flow methods: a comparision of approaches. Regulated Rivers: Research \& Management 13(2), 115 $-127$

Martínez Capel, F. (2011). Modelos de idoneidad de microhabitat para el estudio del régimen ecológico de caudales. Artículo interno Departamento de Ingeniería Hidráulica y Medio Ambiente, Universidad Politécnica de Valencia, España
Mendoza, M. (2015). Dinámica de los procesos hidrológicos en la cuenca del río Nuble en San Fabián. Proyecto de título, Universidad Católica de la Santísima Concepción, Chile

MinEnergía (2016). Nuevo sistema de pronóstico para ERNC. Ministerio de Energía, Chile, 29 de agosto 2016, www.energia.gob.cl/ tema-de-interes/presentan-sistema-de-pronostico

MinEnergía (2015). Bío Bío la capital energética de Chile. Ministerio de Energía, Gobierno de Chile, 30 de noviembre 2015, www. energia.gob.cl/tema-de-interes/biobio-la-capital-energetica-de

MMA (2012). Reglamento para determinación del caudal ecológico mínimo. Decreto 14. Ministerio de Medio Ambiente, Gobierno de Chile

Mosley, M.P. (1983). Flow requirements for recreation and wildlife in New Zealand rivers - a review. Journal of Hidrology New Zealand 22(2), 152-174

Reiser, D.W., Ramey, M.P., Beck, S., Lambert, T.R. and Geary, R.E. (1989). Flushing flow recommendations for maintenance of salmonid spawning gravels in a steep, regulated stream. Regulated Rivers: Research \& Management 3(1), 267 - 275

Schneider, M., Noack, M., Gebler, T. and Kopecki, I. (2010). Handbook for the habitat simulation model CASiMiR. Module CASiMiR-Fish. Base Version. Schneider \& Jorde Ecological Engineering $\mathrm{GmbH}$ and University of Stuttgart Institute of Hydraulic Engineering, Germany

SEA (2016). Guía metodológica para determinar el caudal ambiental para centrales hidroeléctricas en el SEIA. Servicio de Evaluación Ambiental, Chile.

SEA (2017) Guía para la descripción del área de influencia. Servicio de Evaluación Ambiental, Chile.

USACE (2012). HEC-RAS, River Analysis System. Hydraulic reference manual. US Army Corps of Engineers, Davis, CA, USA 


\section{Obras y Proyectos guidelines for authors}

1. The articles submitted should be original and not have been published before nor be in a revision process for other publication. Once the article is received complying with the points detailed below, it will be sent to external reviewers, experts in the specific subject of the article. The authors are responsible for recognising any conflict of interest which might bias the work, for example, naming sources of funding and support.

2. Articles should not have more than 8000 words. No specific format is obligatory, but it is suggested the use of letter size 12 , Times New Roman letter type and double spacing. It is required to write with clarity, which means to follow a logical sequence of what is written. It is also needed clear figures, plots, tables and pictures.

3. Articles should include in the first page the title, name and surname of the authors as well as the affiliation, postal and electronic address of all the authors. The title has to be written in Spanish and English.

4. The abstract should not exceed more than 250 words and should address the key points of the articles and gives and brief resume of the application and main article conclusion. The abstract has to be a correct translation from the Spanish. Keywords have to be included in English and Spanish.

5. The references have to be complete and correct. The references cited in the text, figures and tables have to be included in the reference list at the end of the article and vice versa. In the text, figures and tables, references should be written as Martínez (2008) or (Goodell and Warren, 2006). For more than two authors it should be cited as (Van Abeelen et al., 2008). If the same authors cited have more than one publication the same year, it should be used 2010a, 2010b, etc. The style of the references should consider the following examples:

Moffat, R., Correia, N. and Pastén, C. (2016). Comparison of mean shear wave velocity of the top $30 \mathrm{~m}$ using downhole, MASW and bender elements methods. Obras y Proyectos 20, 6-15

Escribano, D.E. and Nash, D.F.T. (2015). Changing anisotropy of $G_{0}$ in Hostun sand during drained monotonic and cyclic loading. Soils and Foundations 55(5), 974-984

Kausel, E. (2017). Advanced structural dynamics. Cambridge University Press

6. Tables and formulae (equations) should be numbered sequentially in the same order as they appear in the text, with Arabic numbers and referring to them as: Table 1, Table 2, formula (1), formula (2)...etc., as appropriate. Tables should not repeat results presented in plots and the titles for tables put above the table. The unit system accepted is the International System IS.

7. Figures can include plots, sketches and diagrams. Figures should be sent ready for publication, laser print quality. Photographies and pictures should also be of high quality. Sequentially numbered as Figure 1, Figure 2 or Photography 1, Photography 2, etc. and the title for the figure put below the figure. Figures have to be mentioned in the text before they appear.

8. Opinions stated in the articles published in Obras y Proyectos are exclusive responsibility of the authors and they do not reflect necessarily the point of views of the Department of Civil Engineering of the Catholic University of Concepción, UCSC.

9. The submission of an article to Obras y Proyectos implicates that the authors accept to transfer the authors' rights of their articles to the UCSC only if the article is accepted for publication. The copyrights cover the exclusive rights for the reproduction and distribution of the article, including reprints, photographic reproductions, microfilms, scanning or any other similar production method as well as translations. Permissions to use figures which do not belong to the authors have to be obtained by the authors before submitting the article. 


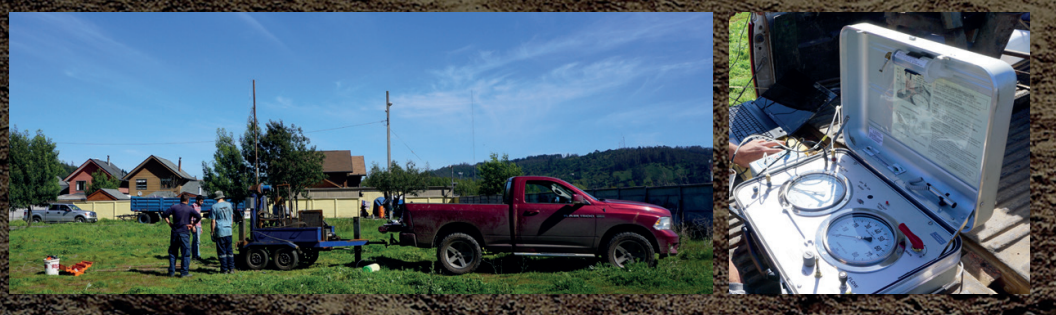

\section{0 años}

alservicio dela región y el país

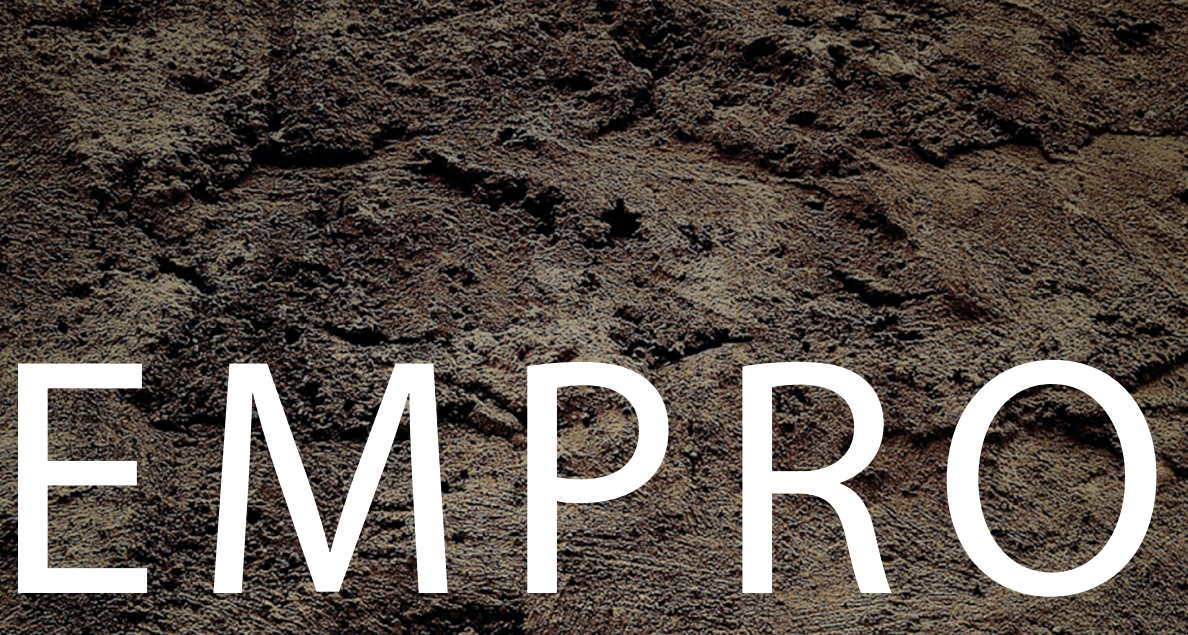

Ensayos de Materiales y Prospecciones

SUELOS - HORMIGONES - ASFALTOS

Ingenieria

Mecánica de Suelos

Fundaciones

Taludes

Mejoramiento de Terreno
Control de Obras Civiles

Densidad in Situ

Hormigones

Asfalios

\section{Prospecition de suelos}

Sondajes Maritimos.

Sondajes Terrestres

Calicatas
Leboratorio Acreditado bajo NCh $15017025 y$ registro vigente MINVU

\section{tris}

